\title{
Global Analysis Peak Fitting Applied to EELS Images
}

M.H. Van Benthem, P.G. Kotula, M. Marinella, W. Mook, and K.L. Jungjohann

Sandia National Laboratories, PO Box 5800, MS 0886, Albuquerque, NM 87185-0886, USA

Spectroscopic transitions often result in features that lend themselves nicely to peak fitting techniques.[1-3] However, just as often, neighboring peaks or background signals obstruct those peaks. Hyperspectral imaging, as opposed to single spectrum collection, may provide some relief to this problem by isolating sources of signal within the image. Employing the common practice of summing or integrating the image[3], or areas of the image, may sacrifice the advantages gained from imaging. Analyzing all of the pixels within the image simultaneously, or globally, permits one to retain the benefits imaging provides throughout the process of analysis and interpretation. In this presentation, we will demonstrate how to perform global peak fitting on STEM-EELS data in an attempt to elucidate the behavior of a Ta/TaOx/Pt stack sputter deposited on a polished W surface, Fig. 1, when challenged with low voltage biasing.

We imaged the sample before, during and after the challenge. We employed a FEI Company Titan G2 80-200 TEM/STEM operated at $200 \mathrm{kV}$ and equipped with a high-brightness Schottky field emission electron source, a spherical aberration corrector on the probe forming optics (AC-STEM) and a Gatan Quantum 963 EELS. The STEM acquired full EELS spectral images (SI) of the low-loss energy region (-20 eV to $180 \mathrm{eV}$ ), including the zero-loss peak, plasmons and $\mathrm{Ta}_{2,3}$ edge, with a 50 msec dwell time.

EELS-SI data preprocessing involved aligning the energy baseline to a common axis, correcting for small instabilities in the primary beam energy and drift/instability of power supplies. We aligned the peaks by first restricting the data set to $-3 \mathrm{eV}$ to $+3 \mathrm{eV}$ (the region of the zero loss peaks). Then we upsampled data by fifty times to an effective channel size of $2 \mathrm{meV}$, using the Piecewise Cubic Hermite Interpolating Polynomial (PCHIP) function in Matlab[4]. Next, we computed the cross-correlation coefficients across the zero loss peaks for each pixel spectrum relative to the first row, first column pixel of the before-challenge sample. Choosing the index of the maximum cross-correlation coefficient as the zero loss value, we shifted all the spectral to a common energy-loss baseline (Fig 2(l)).

The concept of global analysis[5] for these data seeks to fit a set of peaks or transitions using nonlinear optimization to determine parameters such as center and width, then using linear least squares to find the linear amplitude terms. Thus, the data in n-pixel by m-spectral channel matrix $\mathbf{D}$ follows the model

$$
\mathbf{D}=\mathbf{A} \mathbf{S}^{\mathbf{T}}
$$

where $\mathbf{A}$ is the n by p-peak matrix of amplitudes or abundances and $\mathbf{S}$ is the $\mathrm{m}$ by p matrix of unit height peaks. Here the model estimates the columns of $\mathbf{S}$ in a nonlinear sense and linear least square provides the amplitudes as

$$
\mathbf{A}=\mathbf{D S}\left(\mathbf{S}^{\mathrm{T}} \mathbf{S}\right)^{-1}
$$

We employed global analysis on the energy-aligned data fitting five Voigt peaks, two asymmetric Voigt peaks, two edge transitions, and an offset or bias. One of the fit peaks and its spatial extent for the three 
challenge states is displayed in Fig 2(r).

References:

[1] Stöhr, J. “NEXAFS Spectroscopy.” Berlin: Springer, (1992).

[2] Reed, B.W., et al. Phys. Rev. B 60(8), (1999). 5641-5652

[3] Varela, M., et al. Phys. Rev. B 79(8), (2009). 085117.

[4] MATLAB. Natick, MA: The MathWorks, Inc. (ver 2014a).

[5] Beechem, J.M. Global Analysis of Biochemical and Biophysical Data. In Numerical Computer

Methods, L. Brand and M. L. Johnson (Eds.), pp. 37-54. San Diego: Academic Press, (1992).

[6] Sandia National Laboratories is a multi-program laboratory managed and operated by Sandia Corporation, a wholly owned subsidiary of Lockheed Martin Corporation, for the US Department of Energy’s National Nuclear Security Administration under contract DE-AC04-94AL85000.

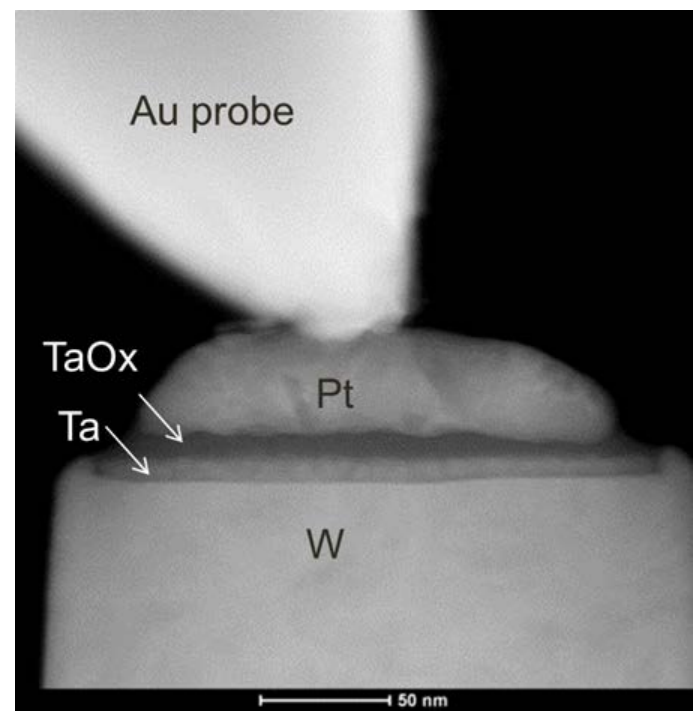

Figure 1. $\mathrm{Ta} / \mathrm{TaOx} / \mathrm{Pt}$ stack on $\mathrm{W}$ surface prepared for STEM-EELS imaging.
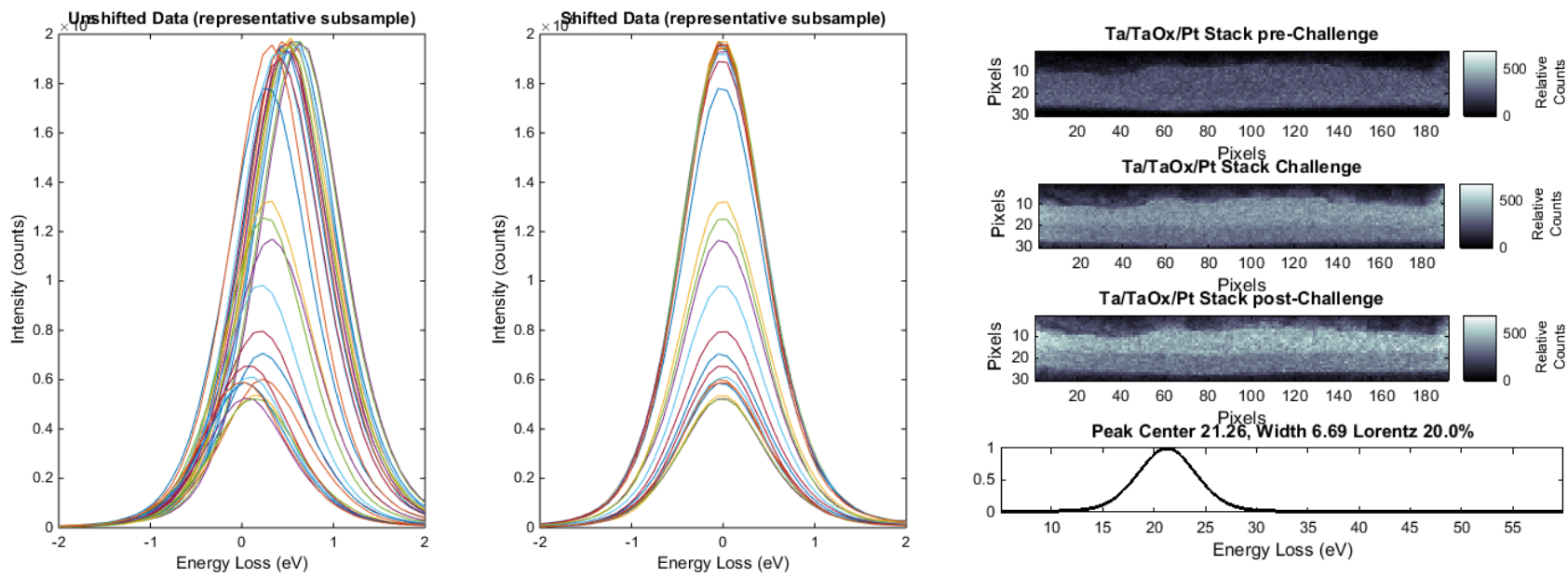

$\mathrm{Ta} / \mathrm{TaOx} / \mathrm{Pt}$ Stack pixels post-Challenge
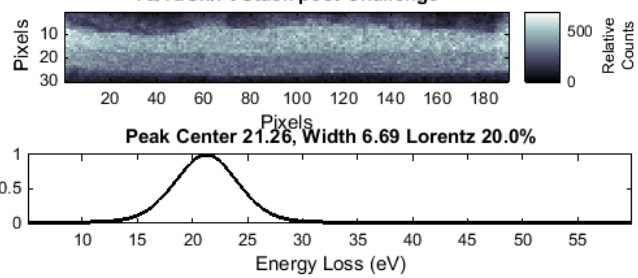

Figure 2. Left: Effects of alignment procedure on EELS-SI zero loss peaks for a small subset of data. Right: Global analysis Voigt peak fit representing notional Ta energy loss from Ta/TaOx/Pt stack. 